\title{
Meeting the challenges of micronutrient deficiencies in emergency-affected populations
}

\author{
Z. Weise Prinzo* and B. de Benoist \\ Department of Nutrition for Health and Development, World Health Organization, 1211 Geneva 27, Switzerland
}

\begin{abstract}
Micronutrient deficiencies occur frequently in refugee and displaced populations. These deficiency diseases include, in addition to the most common Fe and vitamin A deficiencies, scurvy (vitamin $\mathrm{C}$ deficiency), pellagra (niacin and/or tryptophan deficiency) and beriberi (thiamin deficiency), which are not seen frequently in non-emergency-affected populations. The main causes of the outbreaks have been inadequate food rations given to populations dependent on food aid. There is no universal solution to the problem of micronutrient deficiencies, and not all interventions to prevent the deficiency diseases are feasible in every emergency setting. The preferred way of preventing these micronutrient deficiencies would be by securing dietary diversification through the provision of vegetables, fruit and pulses, which may not be a feasible strategy, especially in the initial phase of a relief operation. The one basic emergency strategy has been to include a fortified blended cereal in the ration of all food-aid-dependent populations (United Nations High Commissioner for Refugees/World Food Programme, 1997). In situations where the emergency-affected population has access to markets, recommendations have been to increase the general ration to encourage the sale and/or barter of a portion of the ration in exchange for locally-available fruit and vegetables (World Health Organization, 1999a,b, 2000). Promotion of home gardens as well as promotion of local trading are recommended longer-term options aiming at the self-sufficiency of emergency-affected households. The provision of fortified blended foods in the general ration has successfully prevented and controlled micronutrient deficiencies in various emergency settings. However, the strategy of relying only on fortified blended foods to prevent micronutrient deficiencies should be reviewed in the light of recurring evidence that provision of adequate supplies of these foods is often problematic. Donor policies on the bartering or exchange of food aid should also be clarified. Furthermore, the establishment of micronutrient surveillance systems, including standardized micronutrient deficiency diagnostic criteria, are vital for the control of micronutrient deficiency diseases.
\end{abstract}

Micronutrient deficiencies: Emergencies: Refugees: Adequacy of rations

Outbreaks of micronutrient malnutrition have occurred frequently in refugees and displaced populations who are dependent on international emergency food aid. In the initial stages of an emergency, relief focuses on preventing starvation and protein-energy malnutrition. Food rations attempt to provide mean daily energy requirements, but often no provision is made for micronutrient content, which will inevitably lead to deficiencies.

The problem of outbreaks of micronutrient malnutrition such as scurvy, pellagra and beriberi, once thought to have been eliminated in the world, was intensively discussed in the International Conference on 'Nutrition in times of disaster' in 1988. It became evident that it was not only the much-debated energy content of the rations which was cause for concern, but the micronutrient quality of the ration needed to be a further priority. Since then, there have been several international meetings highlighting the problem of micronutrient deficiencies in refugees and displaced people, and discussions on ways to address the problem, e.g. the Workshop on Improvement of the Nutrition of Refugees and Displaced People in Africa, held in Machakos, Kenya in 1994 (Applied Nutrition Programme, 1995), and the Workshop on Enhancing the nutritional quality of relief diets, held in Washington, DC, USA in 1999 (The FANta Project, 1999). Some examples of reported micronutrient deficiencies among refugees

Abbreviation: IDP, internally-displaced people.

*Corresponding author: Dr Zita Weise Prinzo, fax +4122 7914156, email weiseprinzoz@ who.ch 
Table 1. Reported micronutrient deficiencies among refugees dependent on food aid (data from Berry-Koch et al. 1990; Upadhyay, 1998; World Health Organization, 1990a,b, 2000; Stevens et al. 2001)

\begin{tabular}{ll}
\hline Type of micronutrient deficiency & Countries or regions \\
\hline Fe-deficiency anaemia & Somali refugees in Ogden, Ethiopia 1986 and 1987 \\
& Burundian refugees in Tanzania 1998 \\
& Bhutanese refugees in Nepal 2000 \\
Vitamin A deficiency (xerophthalmia) & Eastern Sudan 1984 and 1987 \\
Thiamin deficiency & Karen refugees in Thailand 1992 \\
& Bhutanese refugees in Nepal 1994-5 \\
Riboflavin deficiency & Kenya (internally displaced) 2000 \\
Vitamin C deficiency & Bhutanese refugees in Nepal 1999 \\
& Kassala Sudan 1991 \\
Niacin deficiency & Kenya 1994 and 1995 \\
& Mozambican refugees in Malawi 1990 and 1991 \\
\hline
\end{tabular}

and internally-displaced people (IDP) are summarized in Table 1.

Emergency-affected populations often live in difficult conditions, making them vulnerable to micronutrient malnutrition. Refugees and IDP often arrive in camps in a nutrient-depleted state, and many are fully dependent on emergency food rations. Such populations often have little access to local markets and, if they do, may have little purchasing power to buy micronutrient-rich foods to supplement their diet. In addition, the growing of vegetables and fruit is often limited by land and water availability.

\section{Micronutrient deficiencies in emergencies}

\section{Vitamin A deficiency}

Vitamin A deficiency is endemic in many parts of South Asia and Africa. It is the main cause of preventable blindness in childhood and is associated with an increased risk of morbidity and mortality due to infectious diseases in young children, and most probably in pregnant women. The main risk factors for becoming vitamin $\mathrm{A}$ deficient in emergencies are: low availability of vitamin A-rich foods; low body stores; decreased breastfeeding (breast milk is a rich source of vitamin A). The extra stress of infections such as measles and diarrhoea, common in emergency-affected populations, can precipitate severe vitamin A deficiency with xerophthalmia within a few weeks, especially if the child is already deficient.

Supplementation is the quickest way to correct vitamin A deficiency, and the most feasible approach to implement. For this reason it is currently the most commonly recommended method of preventing vitamin A deficiency in situations where vitamin A deficiency is prevalent and/or dietary intake is inadequate. At the same time infectious diseases need to be controlled. Dietary sources of vitamin A in the general food ration should be provided when available. Many varieties of fresh fruit and vegetables are good sources of vitamin A, and red palm oil is a rich source of the vitamin. There should be efforts enabling the population to have access to vitamin A-rich foods (e.g. through local markets and/or kitchen gardens). Breastfeeding infants and young children should be promoted and supported in all situations. Fortified commodities such as fortified oil, dried skimmed milk or fortified blended food (cereal-legume mix) should be provided. For example, a ration of $60 \mathrm{~g}$ blended food/person per $\mathrm{d}$ would cover the recommended daily allowance of children under 5 years and about $60 \%$ of the recommended daily allowance for adults.

\section{Iron deficiency and anaemia}

Fe deficiency is probably the most prevalent micronutrient deficiency in emergencies. Fe-rich foods are seldom present in the cereal-based relief diets. In addition, lack of vitamin C in the diet and the high plant-fibre content of the ration all contribute to decreased $\mathrm{Fe}$ absorption. Fe deficiency and its main consequence, anaemia, affects especially women of child-bearing age and contributes to the high maternal mortality rates. It is also common in infants (especially lowbirth-weight infants) and young children, where anaemia is often the result of the combination of several factors: $\mathrm{Fe}$ deficiency; parasitic infections (hookworms, schistosomiasis or malaria); nutritional deficiencies (folic acid, vitamins $\mathrm{B}_{12}$, $\mathrm{A}$ and protein-energy malnutrition).

Provision of adequate dietary sources of $\mathrm{Fe}$ is often not feasible, taking into consideration the poor absorption of non-haem dietary Fe. It is recommended, therefore, that all pregnant and lactating women be given $\mathrm{Fe}$ and folate supplements. Supplementation programmes need to be accompanied by intensive nutrition education and supervision, since compliance with regular daily supplements is usually poor. In addition, the inclusion of fortified blended food in the general ration would be one way of increasing the Fe content of the food basket. Moreover, more general public health measures, including control of parasitic infections, control of malaria and control of other nutritional deficiencies, should be integrated into interventions for Fe-deficiency anaemia control.

\section{Vitamin C deficiency}

It is difficult to meet vitamin $\mathrm{C}$ requirements through the standard emergency ration of cereals, beans and oil. Vitamin $\mathrm{C}$ is mainly found in fresh vegetables and fruit, and is quite 
unstable in foods. Epidemics of scurvy have occurred in the drought- and famine-affected refugee populations in the Horn of Africa (Ethiopia, Sudan, Somalia and Kenya), where refugees have not had access to fresh fruit and vegetables (Magan et al. 1983; Centers for Disease Control, $1989 a, b$; Desenclos et al. 1989). One of the main risk factors is the duration of stay in a refugee camp when the population is dependent on the emergency food ration, reflecting the time on rations lacking vitamin $\mathrm{C}$. Another risk factor is overcrowded camps, where infectious diseases spread quickly, thereby increasing vitamin $\mathrm{C}$ requirements. Low intakes of fresh vegetables and fruit, leading to vitamin $\mathrm{C}$ deficiency, are generally due to: the absence of, or greatly reduced access to, a local market and/or no purchasing power to buy fresh fruit or vegetables; limited possibilities for growing vegetables due to scarce land and water resources, as well as the dry season, when the highest incidences of scurvy usually occur. While scurvy might affect both children and adults, studies in emergencies have shown that the risk is higher in women, especially pregnant women, than men and increases with age (Centers for Disease Control, 1989a,b; Desenclos et al. 1989).

The principal way of addressing vitamin $\mathrm{C}$ deficiency is by improving the diet (World Health Organization, 1999a). However, securing an adequate diet for large emergencyaffected populations can be a problem, especially in the initial phase of a relief operation. One of the most effective ways to enable emergency-affected populations to cover their vitamin $C$ requirements is to support the sale and/or barter of a portion of the ration in exchange for locallyavailable fruit and vegetables (where available). The local cultivation of vegetables such as tomatoes, peppers, onions and leafy vegetables and tubers such as potatoes and sweet potatoes (Ipomoea batatas) should be strongly promoted from the beginning. The drawbacks are often lack of access to land and adequate water supply, and even under optimal conditions it may take 2-3 months for the natural sources of vitamin $\mathrm{C}$ to be ready for consumption. There is, therefore, a need for an alternate intervention strategy. The fortified cereal-legume foods are suitable foods for distribution during the initial emergency phase. Fortification of other foods with vitamin $\mathrm{C}$ still needs to be looked at. When fresh fruit and vegetables are not available, vitamin $C$ can be obtained by germinating pulses or cereals (where accepted and where feasible). In situations where a population is at high risk of scurvy, or where cases of scurvy have already been identified and all the other options for intervention are not immediately feasible, distribution of vitamin $\mathrm{C}$ tablets needs to be considered. It is, however, difficult to maintain consumption consistently over long periods and to achieve good coverage.

\section{Niacin deficiency}

Pellagra caused by deficiency of niacin or its precursor tryptophan is another disease that has shown alarming prominence in refugee and IDP populations where maize is the staple cereal and where beans or groundnuts are not adequately available. While affecting both adults and children, women have been at far higher risk than men, and risk increases with age (Moren \& Le Moult, 1990; Centers for Disease Control, 1991). Pellagra has been rare in infants and in young children.

The main ways of preventing the onset of pellagra in emergency situations affecting large populations are by: provision of food rations containing adequate amounts of bioavailable niacin by diversifying the general ration (a maize-based diet should always contain legumes, meat or fish where available); provision of a second staple (e.g. rice, millet); provision of surplus food in the ration to allow the affected population to sell or exchange for another food commodity; cultivation of home gardens and/or land cultivation, and the keeping of domestic poultry; the addition of a fortified cereal-legume blend to the general ration or the fortification of maize meal with niacin; the provision of niacin in the form of tablets (vitamin B complex) for prevention and treatment of pellagra if the other approaches fail (Toole, 1992; World Health Organization, 2000).

\section{Thiamin deficiency}

Beriberi is most common in emergency-affected populations where polished rice, highly-milled cereals or starchy staples such as cassava and tubers are the principal component of the diet. Similar to vitamin $\mathrm{C}$, thiamin is not very stable in food, and losses can be high depending on the way the food is prepared and cooked (e.g. washing rice before cooking and discarding cooking water). Beriberi can develop within 2-3 months of inadequate consumption of thiamin (Brin, 1963). Anti-thiamin factors are present in, for example, tea and in betel (Areca catechu)-nuts, and can have a direct influence on the development of thiamin deficiency, especially in populations where thiamin intake is already marginal (Combs, 1992). Nutrition education and information campaigns are necessary to modify food habits in those populations where the reduction in intake of anti-thiamin factors can make a difference to the thiamin status of the population. There are increased requirements during pregnancy and lactation. Infants breast-fed by women having deficient thiamin levels can develop infantile thiamin deficiency. This disease is acute, and case fatality is very high. For example, cases of infantile beriberi and thiamin deficiency in pregnant women were reported in 1992 among the Karen refugee population in Thailand (Médecins sans Frontière/Epicentre, 1992).

The main ways of preventing thiamin deficiency in emergency situations affecting large populations are by (World Health Organization, 1999b): increasing the variety of the food basket and regularly including adequate amounts of legumes and vegetables; provision of parboiled rice (the organoleptic qualities of parboiled rice can vary greatly from polished rice; therefore, a strong education programme is vital for the success of such an intervention), under-milled rice or other under-milled cereals instead of polished rice; provision of sufficient food in the ration to allow refugees and IDP to trade for a more varied diet; fortification of current relief commodities with thiamin or provision of fortified blended cereal-legume food in the general ration; thiamin or vitamin $\mathbf{B}$ complex tablet supplementation. 


\section{Approaches to address micronutrient deficiencies in emergencies}

Micronutrient deficiencies have an impact on mortality and morbidity of a population and, therefore, need to be addressed adequately (Toole et al. 1988). Surveillance systems are not always in place to identify micronutrient deficiencies, and simple case definitions are not available for all the micronutrients. Biochemical tests under emergency conditions are limited. Clinical manifestations reflect a late stage of micronutrient malnutrition and, therefore, cannot be used as indicators to predict the problem. Thus, risk factors should be used as indicators to monitor the risk for micronutrient deficiencies in emergencies.

Since the mid 1990s, diversification of diet and free access to local food markets have been recognized as essential for the provision of micronutrients (Hansch, 1992; Toole, 1992). Fortified cereal-legume blends have been distributed in the general ration (United Nations High Commissioner for Refugees/World Food Programme, 1997). Supplementation has been tried, using tablets or orange-concentrate drinks, but except for vitamin A these interventions have not been very successful in alleviating micronutrient deficiencies (Mardel et al. 1995). Supply of food items through home gardens and by trading has developed little. The reality is that refugees are often prohibited to trade, forage or grow food crops.

\section{Dietary diversification}

The following are just a few examples of the advantages and disadvantages of trying to diversify the general ration (Berry-Koch et al. 1990; World Health Organization, 1999a).

Advantages:

1. local fresh foods allow refugees or IDP to maintain a normal diet. Many varieties of fresh fruit and vegetables will provide adequate quantities of carotene, $\mathrm{Fe}$, vitamin $\mathrm{C}$ and thiamin. Lentils and groundnuts provide $\mathrm{Fe}$, thiamin and niacin;

2. natural sources of micronutrients provide other nutrients (trace elements, phytonutrients); local procurement of fresh foods may enhance production by indigenous populations, while providing economic support.

Disadvantages:

1. purchase of sufficient quantities for general distribution has proved difficult and expensive in the past;

2. local markets may be unreliable;

3. cost is usually high;

4. logistic problems, especially if the emergencyaffected population is not readily accessible;

5. large-scale procurement can create shortages and increase prices on the local market to the disadvantage of local consumers.

Feasibility (examples):

1. the limited amounts of citrus fruit, guavas (Psidium guajava) and camel's milk distributed to refugees in
Somalia in 1989 proved to be very difficult logistically and expensive, and this intervention was discontinued (Toole, 1992);

2. vegetables are being distributed to refugees in Nepal. Procurement of sufficient quantities and related logistics seem to be under control. However, Nepal's refugee population of about 85000 is small compared with the millions in Africa, and fresh vegetables are more readily available in Nepal than in the arid area in the Horn of Africa.

Concerning increasing ration size and trade, it has been found that refugees receiving increased rations in fact consume a greater amount of fruit and vegetables; however, it is important to provide information to the households about micronutrient deficiencies and the importance of fruit and vegetables, in order to encourage the regular purchase of such foods.

\section{Fortification}

Blended food. Currently, the most practical alternative to the distribution of fresh foods is providing precooked cereal-legume blends that are fortified. Formulated for use in supplementary feeding programmes for moderately-malnourished children, they are often provided by major donors for distribution in the general ration (Dijkhuizen, 2000). The following are examples of the advantages and disadvantages of using blended food as a means of covering the micronutrient needs of a population (Berry-Koch et al. 1990; World Health Organization, 1999a).

Advantages:

1. blended food is pre-cooked which means minimal losses during preparation;

2. the main nutrients can be provided in one source;

3. the fortified commodity can be kept as a contingency stock for immediate mobilization when required;

4. studies have now shown that it is mostly used for the whole family.

Disadvantages:

1. enhanced coordination of food aid is required to ensure that populations at risk receive blended foods;

2. blended food may not be accepted by all emergencyaffected populations;

3. need to standardize and internationally agree on appropriate micronutrient fortification levels;

4. phytate content might decrease bioavailability of some micronutrients;

5. protein levels may be inappropriate for general distribution;

6. increased dependency on food aid;

7. only a short-term strategy.

Feasibility:

1. Research carried out by Oxfam/United Nations High Commissioner for Refugees looked at the usage of blended foods in an emergency at the household level (Mears \& Young, 1998). There were no major problems 
with either the use or acceptability of blended foods. The study, however, highlighted some technical and operational issues regarding quality control and timely supply of locally-produced food products.

Cereals. The technology exists for fortifying cereal flour with micronutrients, but losses during storage, transport and preparation have to be carefully assessed (Hoffman-La Roche, Basel, Switzerland, personal communication).

Advantages:

1. the fortification of cereals is a convenient way of preventing deficiency diseases by providing food with added micronutrients.

Disadvantages:

1. the shelf-life of fortified milled cereals is reduced compared with unfortified unmilled cereals;

2. monitoring of quality control is essential.

Feasibility:

1. The research carried out by Oxfam/United Nations High Commissioner for Refugees (Mears \& Young, 1998) mentioned earlier showed that cereal fortification involved major issues of technical and operational feasibility (such as milling capacity, site of fortification) which would need to be dealt with for successful implementation of the use of fortified cereals in an emergency. However, a growing number of nonindustrialized countries have embarked successfully on a programme of cereal fortification, especially in Latin America, Asia and more recently Africa (Zambia).

Fortification of other commodities include: salt, which may be fortified with $I$, and there is ongoing experience leading to the addition two or more micronutrients to salt (India); oil is a commodity that is used as a vehicle for vitamin $A$ and vitamin $D$ fortification; fortification of sugar with vitamin $\mathrm{A}$ is carried out in Central America.

\section{Supplementation}

Regular supplementation of specific vitamins (e.g. vitamin C, vitamin B complex) is logistically difficult in large populations affected by emergencies, especially since the tablets need to be taken daily. Routine supplementation is recommended only as a means of treating outbreaks of the deficiency disease (World Health Organization, 1999a,b, 2000). However, vitamin B complex tablets can be distributed to pregnant and lactating women via antenatal and postnatal clinics, in addition to $\mathrm{Fe}$-folate tablets, as a preventive measure.

The distribution of specific micronutrients has the following advantages and disadvantages.

Advantages:

1. supplementation is very specific for treatment and prevention;

2. storage and transportation is less problematic than for foodstuffs;

3. it is a politically-visible form of assistance.
Disadvantages:

1. regular mass distribution and non-compliance are major problems;

2. procurement of sufficient quantities can be difficult;

3. only a short-term measure;

4. Fe and vitamin A supplementation need to be carefully monitored (problem of possible toxicity);

5. does not make up for an inadequate emergency food ration.

\section{Case studies \\ Horn of Africa}

Scurvy in refugee camps in Ethiopia, Somalia and Sudan is an ongoing problem. A study of outbreaks in Somalia in 1982 found that most of those affected were pastoral nomads whose traditional source of vitamin C was camel's milk (Magan et al. 1983). Although the refugees involved in the outbreaks in Somalia had been in camps for as long as 3 years, they had been supplementing their general rations with food (e.g. camel's milk, tomatoes, onions, sweet potatoes) purchased in local markets in and around the camps. At 6 months before the scurvy outbreak, however, the Government of Somalia ordered the closure of all local markets. As a result, poorer refugees were unable to purchase sufficient amounts of camel's milk and other vitamin C-containing foods due to a sharp rise in price, which resulted in the outbreak of scurvy. Vitamin $C$ tablets were distributed weekly to all refugees and increased surveillance measures were instituted.

Persistent outbreaks of scurvy among Somali and Ethiopian refugees have also occurred in Kenya (Stevens et al. 2001). In September 2000 Save the Children Fund (UK) (unpublished results) reported an outbreak of scurvy and suspected beriberi in north-eastern Kenya. Shortages of fruit and vegetables in the markets because of drought, over-cooking of maize-soya milk, and seasonal factors affecting food availability were associated with scurvy in Kenya. Seasonal outbreaks remain despite various strategies of tablet distribution and distribution of fortified foods.

Lessons learnt were:

1. distribution of fortified food to the whole emergencyaffected population may currently be the only option in emergency situations;

2. micronutrient deficiencies in emergency situations often appear as a complex picture of multiple deficiencies complicating assessment and diagnosis;

3 . field personnel need to be trained in diagnosing micronutrient deficiencies;

4. surveillance systems need to be set up to look at all the risk factors mentioned earlier (see p. 253).

\section{Bhutanese refugees in Nepal}

There have been persistent micronutrient problems among refugees in Nepal (Upadhyay, 1998). In 1993 the first cases of beriberi were reported. A surveillance system was then set up by Save the Children Fund (UK), and within a short time thereafter cases of pellagra and scurvy were reported. 
The food basket consisted of polished rice, lentils, oil, salt, sugar and fresh vegetables. Several factors were identified which were likely to result in micronutrient deficiencies, including: distribution of polished rice; distribution of types of vegetables that did not contribute much to micronutrients in the diet (the provision of fresh vegetables was logistically very difficult and it was often not possible to obtain enough variety in bulk); restrictions on ration trading, and unfavourable exchange rates for general ration commodities (the refugees purchased meat and dairy products).

The food basket was then modified in early 1994 as follows; polished rice was replaced by parboiled rice; UNILITHO (locally-produced blended food) was added to the general ration; green or yellow vegetables (rather than radish) were distributed in the vegetable ration; a rigorous nutrition education programme was undertaken to improve the acceptability of parboiled rice and blended food (Upadhyay, 1998; G Clugston, unpublished results). Blended food was initially viewed as a food for the sick, but was gradually accepted for daily consumption as a porridge and consumed with the daily morning tea.

In May 1997 a household food economy assessment carried out by Save the Children Fund (UK) (1997; unpublished results (on behalf of the World Food Programme, Nepal and United Nations High Commissioner for Refugees, Nepal) came to the following conclusions:

1. it is difficult to verify the accuracy of clinical diagnosis of micronutrient deficiency diseases without biochemical assessments;

2. the general food ration was still low in $\mathrm{Ca}$ and riboflavin;

3. intra-household maldistribution of food may have partly explained the continuing low incidence of micronutrient deficiency diseases;

4. storage and cooking practices had reduced the micronutrient content of food consumed.

Lessons learnt were:

1. food rations that are adequate in terms of energy and protein are not necessarily balanced and do not address all the nutritional needs of a population;

2. inclusion of blended food should be mandatory in emergency situations where access to a variety of micronutrient-rich foods is not feasible;

3. food habits of emergency-affected populations should be given due consideration;

4. nutrition education can play an important role in supporting interventions to address micronutrient deficiencies;

5. issues concerning household food distribution, and cooking and storage practices should be looked into and problems addressed.

\section{Ongoing research (examples)}

Analysis of the micronutrient status of populations affected by emergencies poses special problems. For many micronutrients no practical biochemical assessment is currently possible in the difficult circumstances encountered in the field. Among others, the Institute of Child Health, London,
UK is engaged in projects to develop a novel way of detecting a range of vitamin concentrations in dried blood spots (blood-spot technology) which offers important advantages over venous sampling.

The World Food Programme/Oxfam have started a joint initiative to try out a containerized milling unit at refugee camp level (Dijkhuizen, 1998). This unit will allow micronutrient fortification of cereals at camp level. Suitable equipment still needs to be developed and field tested. If successful, this equipment will reduce the need for fortified blended foods in general rations.

United Nations High Commissioner for Refugees/ Institute of Child Health have started operational research to look at the efficacy and effectiveness of using iron pots for cooking to control anaemia in refugees. The idea is that cooking in iron pots will increase the $\mathrm{Fe}$ content of foods and that this strategy might be valuable in helping to reduce the incidence of anaemia in emergency-affected populations.

\section{Conclusion}

In conclusion, the following are the main challenges for meeting micronutrient malnutrition in emergencies in the forthcoming years:

1. develop user-friendly tools to assess the magnitude of micronutrient malnutrition and to identify populations at risk and to monitor the impact of interventions; 2. strengthen surveillance systems to monitor the impact of interventions;

3. enable dietary diversification;

4. develop internationally-agreed micronutrient premix specifications for fortification of food aid commodities.

\section{References}

Applied Nutrition Programme (1995) Report on a Workshop on the Improvement of the Nutrition of Refugees and Displaced People in Africa, Machakos, Kenya, 5-7 December 1994. Nairobi, Kenya: University of Nairobi.

Berry-Koch A, Moench R, Hakewill P \& Dualeh M (1990) Alleviation of nutritional deficiency diseases in refugees. Food and Nutrition Bulletin 12, 106-112.

Brin M (1963) Thiamine deficiency and erythrocyte metabolism. American Journal of Clinical-Nutrition 12, 107-116.

Centers for Disease Control (1989a) Nutritional status of Somali refugees in eastern Ethiopia, September 1988-May 1989. Morbidity and Mortality Weekly Report 38, 455-456.

Centers for Disease Control (1989b) Nutrition and health status of displaced persons-Sudan, 1988-1989. Morbidity and Mortality Weekly Report 38, 848-855.

Centers for Disease Control (1991) Outbreak of pellagra among Mozambican refugees - Malawi, 1990. Morbidity and Mortality Weekly Report 40, 209-214.

Combs GF Jr (1992) The Vitamins: Fundamental Aspects in Nutrition and Health. San Diego, CA: Academic Press.

Desenclos JC, Berry AM, Padt R, Farah B, Segala C \& Nabil AM (1989) Epidemiological patterns of scurvy among Ethiopian refugees. Bulletin of the World Health Organization 67, 309-316.

Dijkhuizen P (1998) Funding to test out camp level milling and fortification equipment. Field Exchange. Special Focus: 
Micronutrients. Emergency Nutrition Network, issue 5, p. 13. Dublin: Emergency Nutrition Network.

Dijhkuizen P (2000) Processed complementary foods in the WFP. Food and Nutrition Bulletin 21, 62-64.

Hansch S (1992) Diet and ration use in Central American refugee camps. Journal of Refugee Studies 5, 300-312.

Magan AM, Warsame M, Ali-Salad A-K \& Toole MJ (1983) An outbreak of scurvy in Somali refugee camps. Disasters 7, 94-97.

Mardel S, Hailey PC, Mahmutovic A, Broom J, Golden MHN, Franklin M \& Mills CF (1995) Micronutrient status of the besieged residents of Sarajevo: May 1993. European Journal of Clinical Nutrition 49, Suppl. 2, 546-561.

Mears C \& Young H (1998) Acceptability and Use of Cereal-based Foods in Refugee Camps-Case Studies from Nepal, Ethiopia and Tanzania. Oxfam Working Paper. London: Oxfam.

Médecins sans Frontières/Epicentre (1992) Study of the food habits of breast-feeding women among the Karen refugee population in the Mae Sod region, Thailand 1992. Medical News 1, 4.

Moren A \& Le Moult D (1990) Pellagra cases in Mozambican refugees (letter). Lancet 335, 1403-1404.

Stevens D, Araru P \& Dragudi B (2001) Outbreak of micronutrient deficiency diseases: did we respond appropriately? Field Exchange. Emergency Nutrition Network, issue 12, pp. 15-17. Dublin: Emergency Nutrition Network.

The FANta Project (1999) Enhancing the Nutritional Quality of Relief Diets. Washington, DC: The FANTa Project, AED.
Toole MJ (1992) Micronutrient deficiencies in refugees. Lancet 339, 1214-1216.

Toole MJ, Nieburg P \& Waldman RJ (1988) The association between inadequate rations, undernutrition prevalence, and mortality in refugee camps: case studies of refugee populations in eastern Thailand, 1979-1980, and eastern Sudan, 1984-1985. Journal of Tropical Pediatrics 34, 218-224.

United Nations High Commissioner for Refugees/World Food Programme (1997) Guidelines for Estimating Food and Nutritional Needs in Emergencies. Geneva: UNHCR (available on request).

Upadhyay J (1998) Persistent micronutrient problems among refugees in Nepal. Field Exchange. Special Focus: Micronutrients. Emergency Nutrition Network, issue 5, pp. 4-5. Dublin: Emergency Nutrition Network.

World Health Organization (1999a) Scurvy and its Prevention and Control in Major Emergencies. World Health Organization Publication. WHO/NHD/99.11. Geneva: WHO.

World Health Organization (1999b) Thiamin Deficiency and its Prevention and Control in Major Emergencies. World Health Organization Publication. WHO/NHD/99.13. Geneva: WHO.

World Health Organization (2000) Pellagra and its Prevention and Control in Major Emergencies. World Health Organization Publication. WHO/NHD/99.12. Geneva: WHO. 